\title{
Aerodynamic Performance Enhancement of Supersonic 2D Missile Using ANSYS
}

\author{
Ashwini Anand Gaonkar, Poornima Menon, Srinivas G." \\ Department of Aeronautical and Automobile Engineering, Manipal Institute of Technology, Manipal Academy of Higher Education
} (MAHE), India

Received October 20, 2019; Revised December 17, 2019; Accepted December 24, 2019

Copyright $\odot 2019$ by authors, all rights reserved. Authors agree that this article remains permanently open access under the terms of the Creative Commons Attribution License 4.0 International License

\begin{abstract}
Missile is a self-propelled vehicle flying at supersonic speeds. Their payloads are usually explosive and are also known as warheads. These warheads are used to destroy a pre-set target. The aim of this paper is to optimize the values of the lift and drag forces on the given missile for better aerodynamic performance, by carrying out numerical simulations over the supersonic missile by varying the angle of attack through a set of suitable boundary conditions, while keeping the Mach number of the missile as a fixed parameter. Aerodynamic performance of the missile is studied by varying the angle of attack from 0 to 12 degrees. For every angle of attack, the coefficient of lift and coefficient of drag variations were studied in detail and were compared with the existing literature survey so as to obtain the maximum value of $C_{L} / C_{D}$ ratio in order to improve the efficiency. A model of the missile was designed to-scale on Space Claim and the flow analysis was done on FLUENT standalone system using ANSYS 16 workbench. The results obtained, were in the form of flow contours of parameters such as velocity, temperature, density, pressure and turbulence. Through these flow contours the maximum and minimum values of all parameters, as well as the variation in these parameters were estimated. From the numerical analysis it was found that maximum value of $C_{L} / C_{D}$ ratio was 2.5 at $12^{\circ}$ angle of attack. It was also found that the velocity increased with increase in angle of attack and increased the efficiency. These results are advantageous upcoming to designers who aim to build aerodynamically efficient missiles.
\end{abstract}

Keywords Aerodynamic, Efficiency, Missile, Numerical Analysis, Performance, Angle of Attack

\section{Introduction}

Missile is a self-propelled weapon often having a type of guidance system and usually carries a payload consisting of explosives known as warheads, capable of causing mass destruction. Missiles are designed in order to support the environmental conditions they are supposed to operate in. There are different types of missiles and these are classified on different basis. Basically the missiles can be either ballistic or cruise. The cruise missiles are further classified on the basis of speed as subsonic, supersonic and hypersonic missiles. The aerodynamic characteristics and design of all these three types of missiles will vary greatly because of the variation in the cruise speed and flight conditions. The subsonic missiles fly at Mach 0.9 and below whereas the hypersonic missile operates above Mach 5 . The body and configuration of different components of the missile are designed and optimized to withstand these supersonic and hypersonic aerothermodynamic conditions. The major mechanisms used in a missile include the Guidance systems, Propulsion systems, Aerodynamic systems and Structural systems which are controlled by the fins, air frame, warhead, etc.

The aerodynamic features, also called the control surfaces of the missile, control the missile flight. The fin and tail are the major steering control surface for the missile. The purpose of a fin is to maintain the stability and orientation of the missile and to avoid deviation from flight path due to perturbation caused during flight. Types of fins include the Planar fins, Grid fins etc. The grid fins, also known as the Lattice fins have been widely used on conventional missiles as its structure is designed to avoid the blockage of the airflow at high supersonic speeds. This makes the functioning of the actuators and the control surfaces easier. Thus such missiles offer lower drag with enhanced performance. Fins offer high maneuverability at high Mach numbers and high angles of attack. Hence fin optimization is a crucial requirement for the efficient performance of a missile. This paper gives more emphasis on the performance study of supersonic cruise missile. 


\section{Literature Review}

Song Tian et al [2], studied the rolling effect of the missile, wherein an angle of attack feedback was provided to the rolling missile through a three loop autopilot system in order to track and observe the dynamic stability of the missile. Hence, it was observed that the angle of attack played a major role in maintaining the stability of the missile. The study mostly involved the mathematical modelling and analytical solving of the problem statement. The results obtained were further verified through numerical methods. Through mathematical derivation it was found that the angle of attack feedback system optimized the missile stability.

Experimental results are the ones obtained by using a scaled down version of the actual model, also known as a prototype. This prototype is placed in a hypersonic wind tunnel or any other similar setup which artificially recreates the operational environment and is also varies certain desired parameters to manually analyze the flow patterns over the body. Experimentation, however, is a cumbersome procedure as it requires the manual recreation of parameters such as supersonic velocity, high altitude density, pressure, etc.

A majority of the flow analysis done on the missile were done through numerical techniques using Computational Fluid Dynamics (CFD) [7][8]. The software generates the calculations based mostly on the Reynolds Averaged Navier-Stokes (RANS) Equations. This is governed by the basic important equations of continuity, momentum, energy and turbulence, taking the viscous effects and boundary layer conditions into account. The results of the calculations generated through these equations lead to the different flow parameter contours over the body. Across all experiments conducted using numerical methods, it was observed that the main motive for using such techniques is to obtain accurate results of new or optimized designs without the need to setup a test rig. Before any analysis could be run, an accurate model had to be designed so as to recreate real conditions and obtain precise results. The standard procedures for all CFD computations began with the creation of an appropriate mesh. This could be either structured or unstructured type of mesh. Following the creation of mesh, the boundary conditions are set based on the working environment of desired object or body [4][5]. Initial calculations are based on the previous experiments already performed so as to provide a reference for further calculations. Hence it is possible to arrive at valid conclusions through the solution graphs and contours obtained.

\section{Methodology}

The missile taken to carry out this study was a standard AGARD-B model of slender, four-fin, blunt-nose configuration whose geometry was taken from [1]. The blunt nose was chosen for the missile so as to make the configuration of the missile aerodynamically efficient by minimizing the shock waves generated at the nose-tip by transferring the kinetic energy generated, to the atmosphere, thus reducing the frictional effects and on the missile body. For different turbulence models and various angles of attack on airfoil studied in detail by Armaan A. and Srinivas G. et al $[9,10]$.

\subsection{Modeling}

The missile [1] was modelled in Spaceclaim using basic sketching tools with a length (L) of $763.7 \mathrm{~mm}$. The creation of the domain was done to analyze the nature of the flow specifically around the missile model and within the domain so as to obtain more accurate results. The domain created for the model was a rectangular extending to $1.5 \mathrm{~L}$ upstream, $3 \mathrm{~L}$ downstream and $2 \mathrm{~L}$ in the cross-stream direction from the missile. After sketching the missile in SpaceClaim as shown in Fig. 1, it was modelled in Design Modeler as in Fig. 2 for surface generation of the 2-D missile and domain. Further, a subtractive Boolean was generated in order to separate the missile surface from the domain surface so as to ensure the flow moves around the missile and not through it.

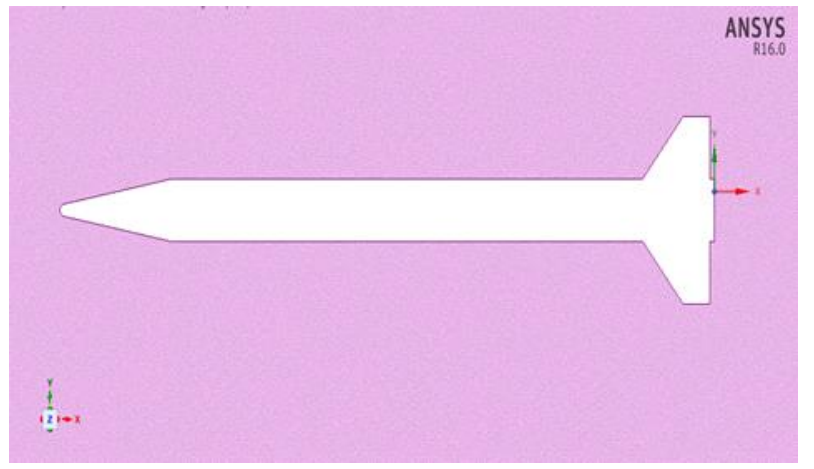

Figure 1. Model of Missile in ANSYS SpaceClaim

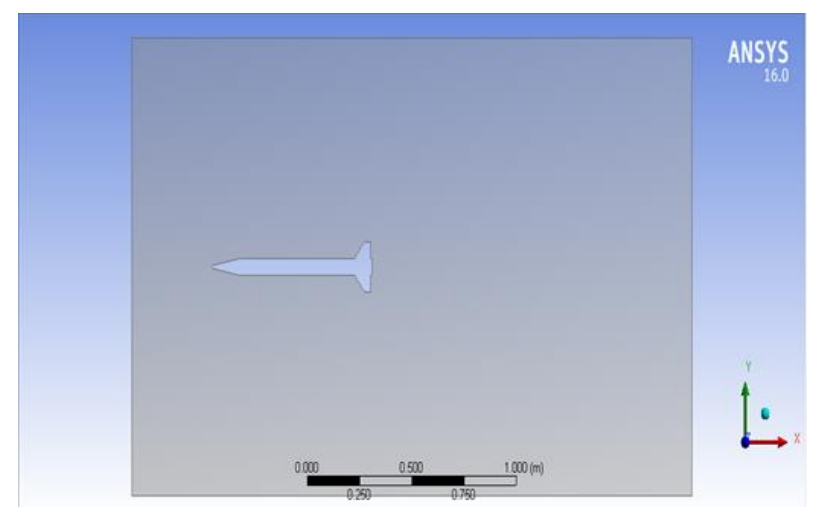

Figure 2. Model of Missile in ANSYS DesignModeler

\subsection{Meshing}

Finite volume approach was used for computing the flow analysis over the missile. It was required to create small 
element within the domain so as to obtain the required average parametric value of the individual elements in the flow field.

An unstructured grid of the rectangular domain was generated with triangular elements, with number of nodes and elements being 388756 and 766806 respectively. Body sizing was given in the form of sphere of influence around the missile body in order to refine the elements near the body within the radius of given sphere. For the missile, edge sizing was given for the individual edges of the 2D missile model by dividing the edges into appropriate number of divisions so that finer elements were created around the body. Since precise flow results were required nearer to the body surface, refinement was given with a factor of 3 . The domain was considered to be a pressure far field and the edges of the missile body were considered to be the walls.

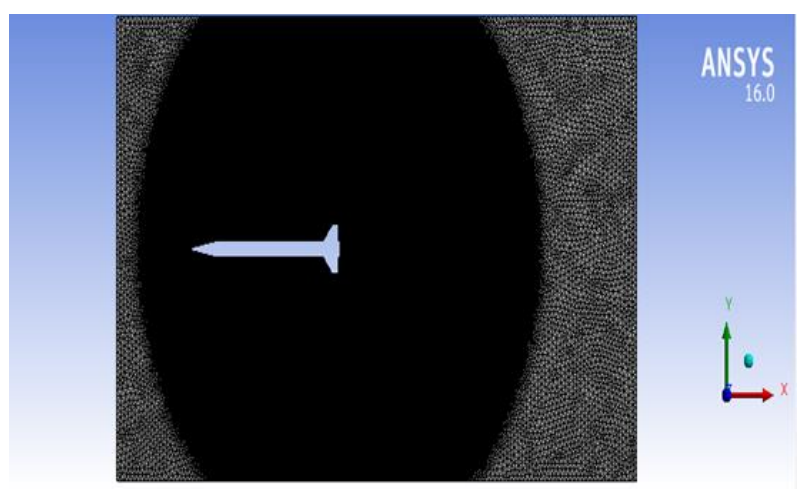

Figure 3. ANSYS Mesh showing sphere of influence.

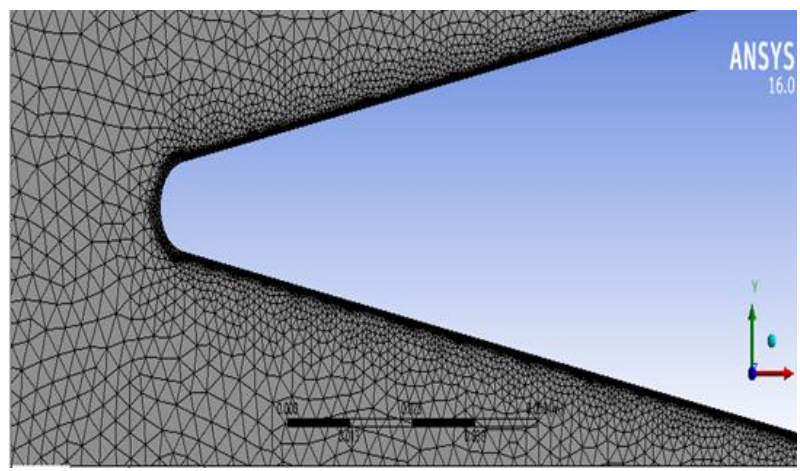

Figure 4. ANSYS Mesh showing refinement around the missile body.

\subsection{Flow Setup}

Since the flow is supersonic, density based solver was used in FLUENT to carry out the flow analysis. The turbulence model used was k-omega SST for better turbulence results and with the energy mode on. The fluid was assumed to be an ideal gas so as to implement the equation of state.

Boundary conditions were set for the pressure far field with respect to the pressure, which was set to be the ambient pressure at an altitude of $10200 \mathrm{~m}$ as mentioned in the reference paper [1]. Mach number was set as a constant parameter throughout the analysis at all the angles of attack with a value of 2.5. This was one of the values taken from the reference paper [1]. The $\mathrm{x}$, y components were set as the horizontal and vertical components, of the given angle of attack, respectively. This angle of attack is taken as a variable parameter ranging from $0^{\circ}$ to $12^{\circ}$, with initial reference analysis being carried out at an angle of attack at $6.05^{\circ}$, which is one of the values from the reference paper [1]. Keeping the specific dissipation rate and turbulent kinetic energy as second order equations the flux type was set as AUSM in order to take the supersonic condition into account. The setup was initialized with reference frame as relative to cell zone. The parameters to be calculated as output results were chosen as lift and drag coefficients. The convergence level was set to be as $10^{-6}$ and the iterations were carried out till the convergence accuracy was reached up to a maximum of $10^{-6}$.

\section{Grid Independence Test}

The grid independence test was carried out for the initial model with an angle of attack of $6.05^{\circ}$. Three different meshes were created for the given model. The number of elements in each of the meshes were 1520481, 766806 and 157326 respectively. This was achieved by varying the maximum and minimum element size of every mesh. Analysis were run using each of these three meshes individually and the best accurate result obtained was from the mesh with number of elements of 766806 and this mesh was further used to carry out the rest of the analysis with the remaining angles of attack.

As inferred from Figure 5 , the closest value of $C_{L} / C_{D}$ value of the grid independence test to that of the reference value is the value obtained from grid independence 2 , and hence having the maximum value of aerodynamic efficiency among the ones obtained from the three grid independence tests. 


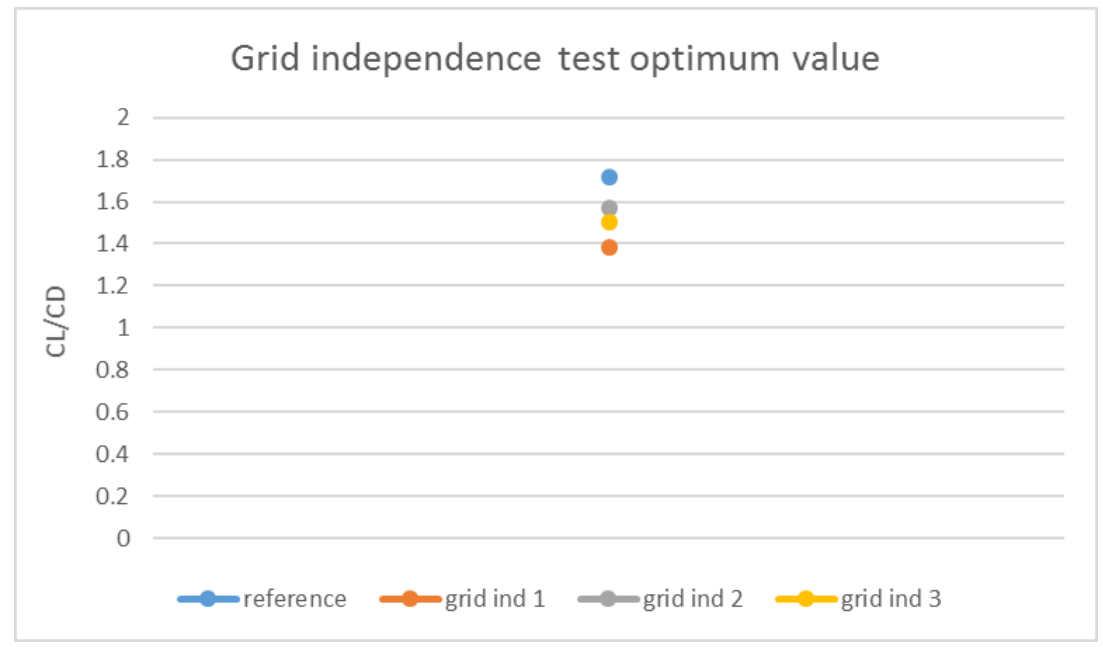

Figure 5. Comparison of grid independence test values

\section{Results and Discussion}

\subsection{Flow Contours}

From the numerical results the flow contours extracted were those of velocity, Mach number, pressure, density, temperature and turbulence.

Fig. 6 to 9 below show the flow contours of velocity, pressure, density and temperature respectively at angle of attack of $6.05^{\circ}$

\begin{tabular}{|c|c|c|c|c|}
\hline Figure & $\begin{array}{c}\text { Angle of } \\
\text { Attack }(\alpha)\end{array}$ & Parameter & Contours & \\
\hline . & $6.05^{\circ}$ & Velocity & 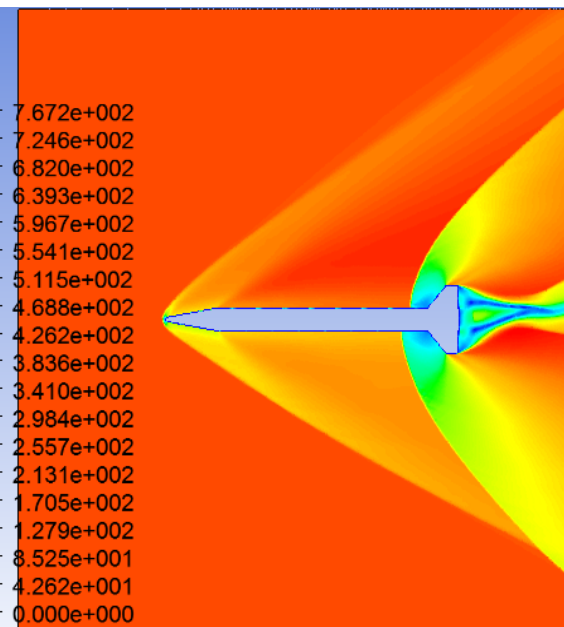 & i \\
\hline
\end{tabular}




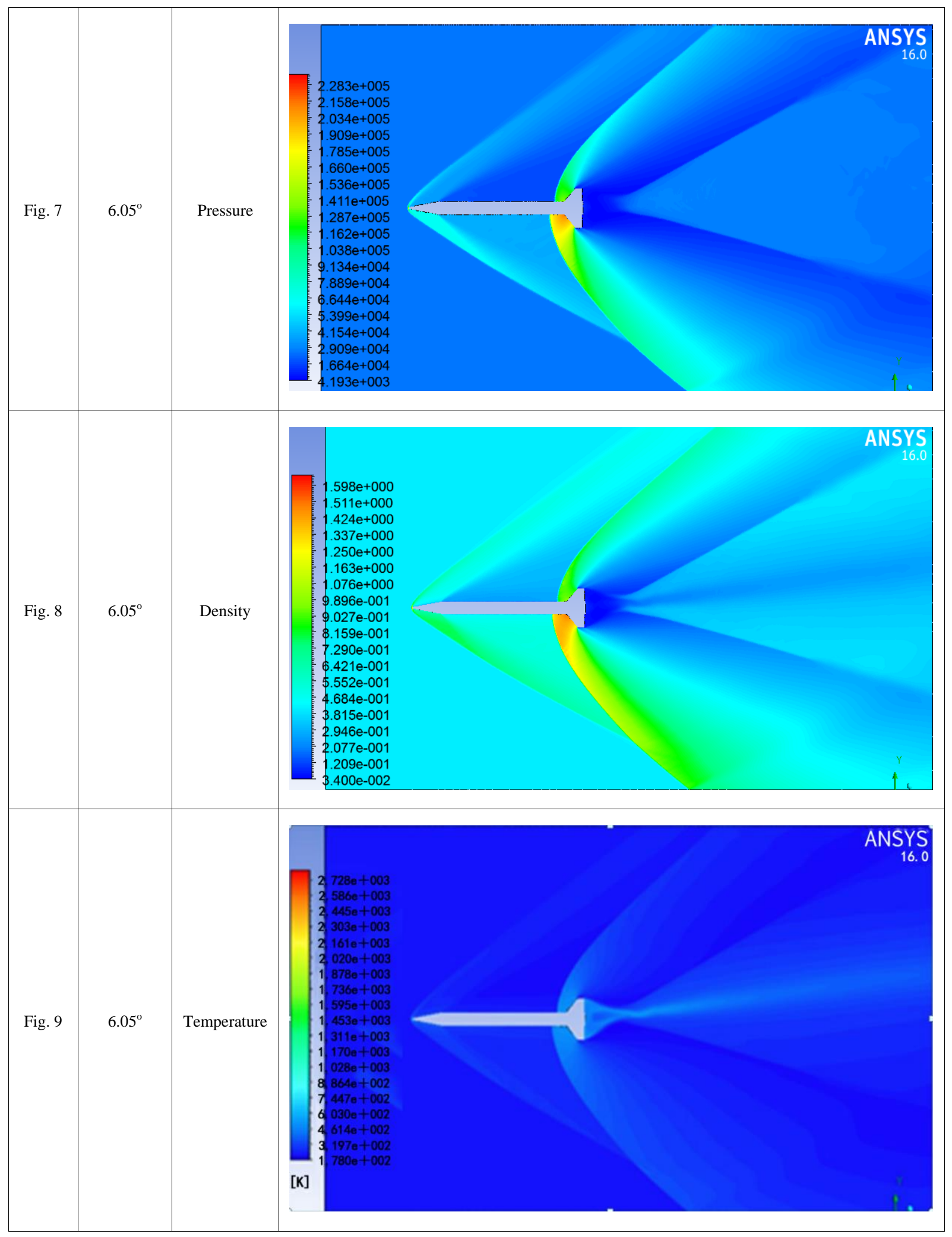

The flow contours along with the $C_{L}$ and $C_{D}$ values were closely studied in order to initially validate the results of angle of attack of $6.05^{\circ}$ and later to check the effect of angle of attack variation on the values of $C_{L}$ and $C_{D}$ so as to obtain an optimum value of angle of attack for maximum value of $C_{L} / C_{D}$, which is the aerodynamic efficiency of the missile.

From Fig 6, which is the velocity flow contour at an angle of attack of $6.05^{\circ}$, it was seen that there is one attached shock generated at the missile nose, as well as a 
normal shock, generated at the tail area of the missile. These shock waves slow down the flow, having very low value velocity behind the normal shock wave. Also, from Fig. 6 to 9, it was observed that the velocity values reduced, whereas the pressure, temperature and density values were found to have increased behind the shocks formed. Ahead of the fins, the flow was seen to be separated from the surface of the missile. This flow separation further saw reduction in the flow properties, having their least values after the flow separation.

Similar Flow patterns was observed when the angle of attack was varied from $0^{\circ}$ to $12^{\circ}$, with the generation of the shock waves and flow separation at the end of the body, with the reduction in the flow parameter value of velocity whereas increase in pressure, temperature and density values as shown in Fig. 10 to 17. At a given point, the flow parameters, however, were noticed to have a greater value in higher angle of attack than in the lower angle.

Figures 10 to 16 show the flow contours of velocity, pressure, density and temperature at angles of attack of $0^{\circ}$ and $12^{\circ}$ respectively:

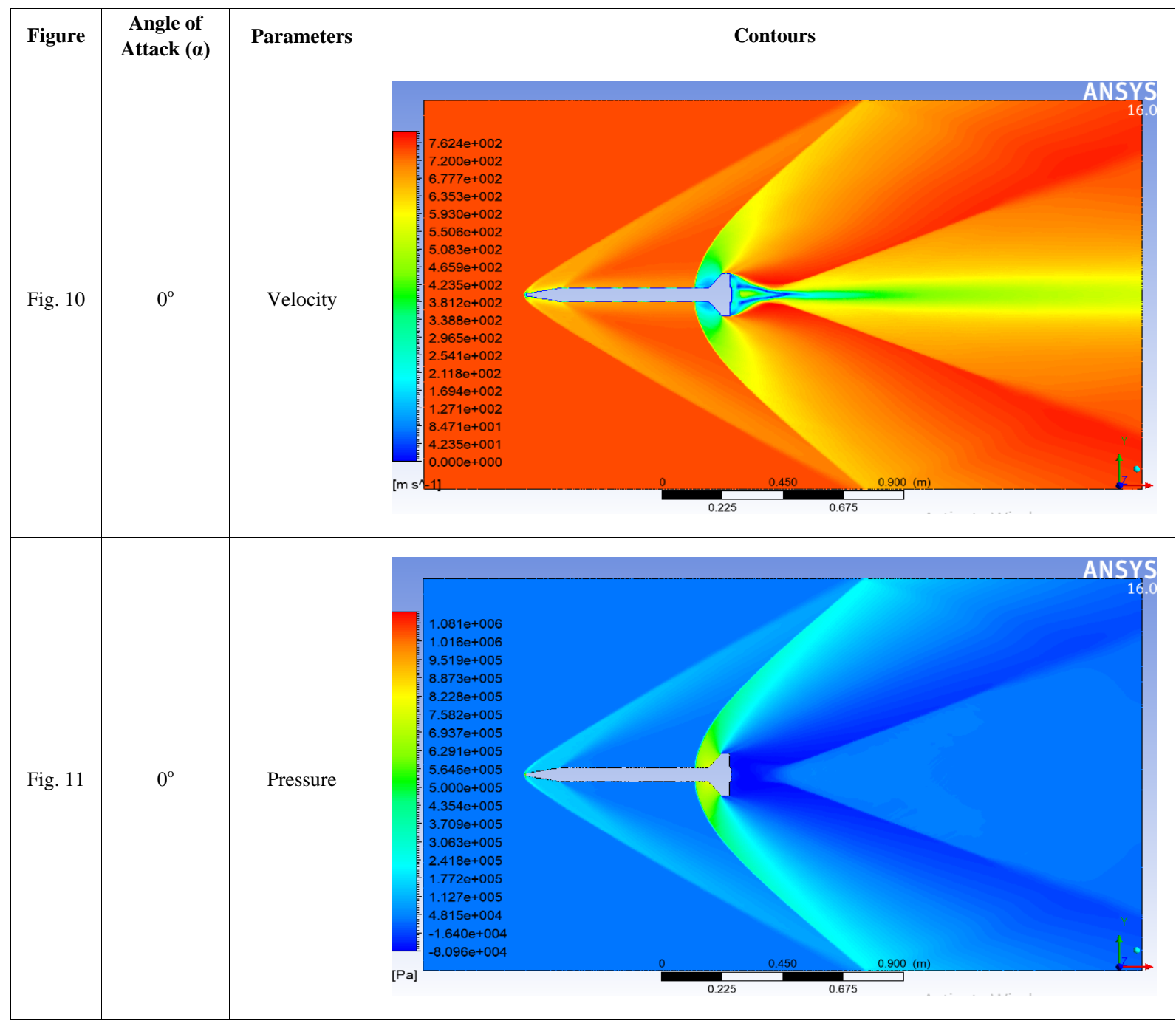




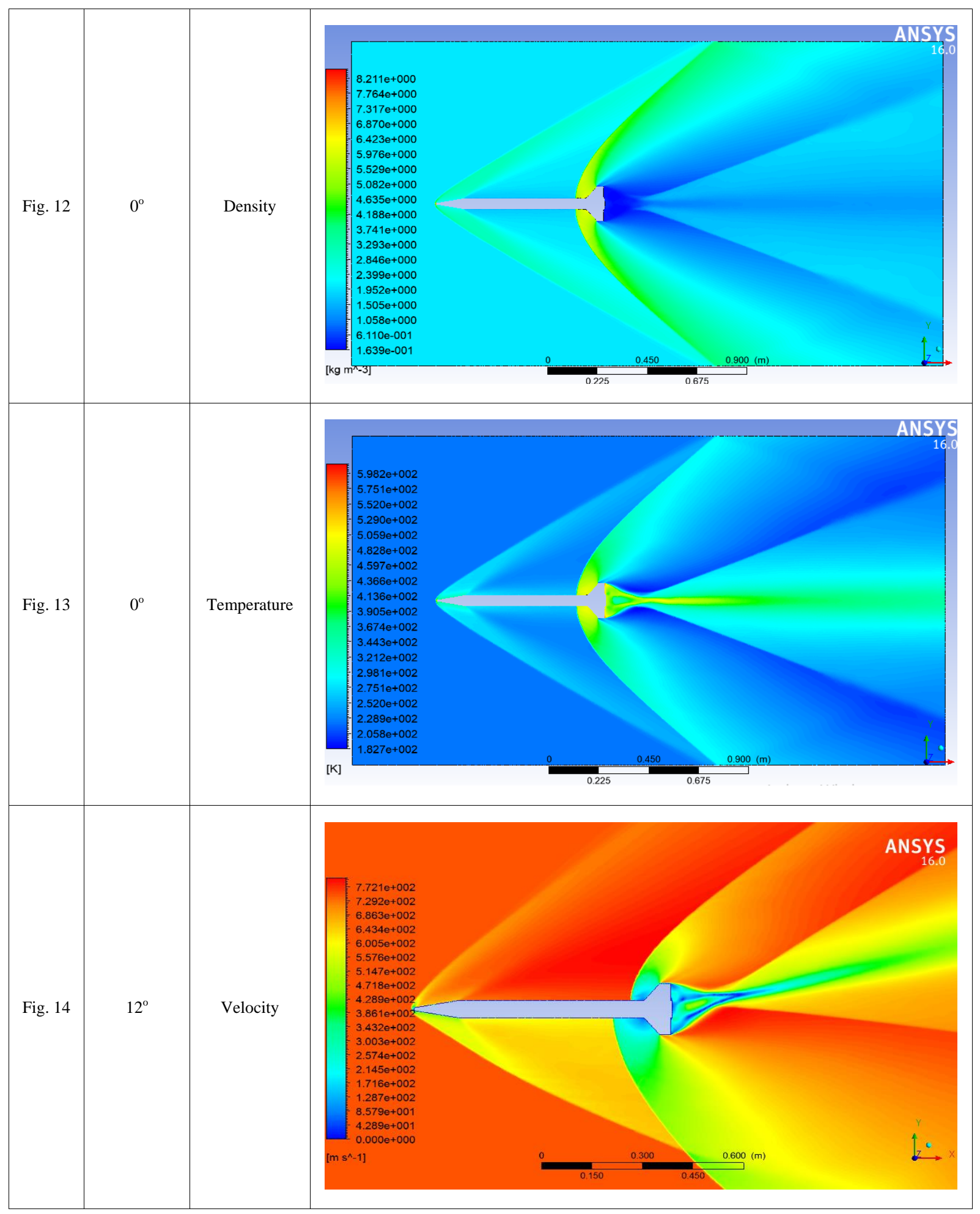




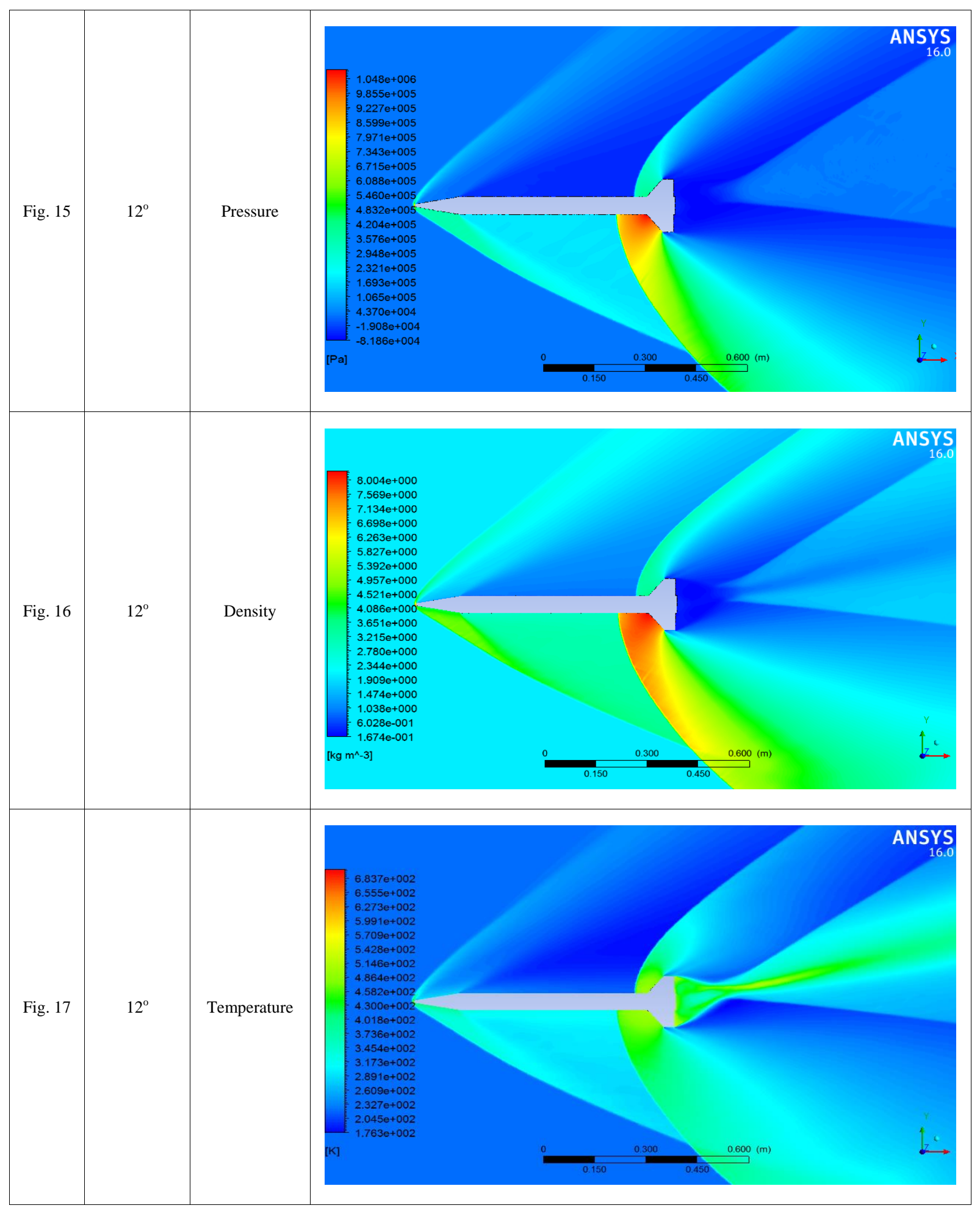




\subsection{Geometry and Meshing}

The boundary made around the missile body [1] was a C-Domain with standard domain dimensions used in most of the research papers. But the domain used in the analysis was a rectangular domain due to incorrect results obtained in the C-Domain due to the improper shock formation at the curved edge due to hypersonic speeds and also the computational time was reduced in a rectangular domain. Also, the meshing used refinement instead of inflation, unlike the reference paper as it yielded better mesh quality.

\subsection{Validation}

The comparison of the $C_{L}$ and $C_{D}$ for the missile at the reference angle of attack of $6.05^{\circ}$ between their values as mentioned in [1] and the calculated ones are listed as follows in Table 1.
Table 1. comparison between reference paper \& obtained numerical values of $\mathrm{C}_{\mathrm{L}} \& \mathrm{C}_{\mathrm{D}}$

\begin{tabular}{|c|c|c|c|c|}
\hline & $\begin{array}{c}\text { Mach } \\
\text { Number }\end{array}$ & $\begin{array}{c}\text { Angle of } \\
\text { Attack }(\alpha)\end{array}$ & $\mathrm{C}_{\mathrm{L}}$ & $\mathrm{C}_{\mathrm{D}}$ \\
\hline $\begin{array}{c}\text { Reference Paper } \\
\text { Values }\end{array}$ & 2.5 & $6.05^{\circ}$ & 1.011 & 0.5875 \\
\hline $\begin{array}{c}\text { Obtained } \\
\text { Numerical } \\
\text { Values }\end{array}$ & 2.5 & $6.05^{\circ}$ & 0.9665 & 0.6157 \\
\hline
\end{tabular}

A series of analysis were carried out with angle of attack ranging from $0^{\circ}$ to $12^{\circ}$ whose theoretical values of $C_{L}$ and $C_{D}$ were calculated using linear interpolation between Angle of attack and $C_{L}, C_{D}$ values from their existing values at $6.05^{\circ}$. The numerical values of $C_{L}$ and $C_{D}$ were also calculated on ANSYS at these angles. The results are stated in Table 2.

With an increment in the value of angle of attack from $0^{\circ}$ to $12^{\circ}$, it was found that both the numerical $C_{L}$ and $C_{D}$ values increased.

Table 2. Variation of $C_{L} \& C_{D}$ with Angle of Attack $(\alpha)$

\begin{tabular}{|c|c|c|c|c|c|}
\hline Mach Number & Angle of Attack $(\alpha)$ & $\mathrm{C}_{\mathrm{L}}($ Theoretical) & $\mathrm{C}_{\mathrm{L}}$ (Numerical) & $\mathrm{C}_{\mathrm{D}}$ (Theoretical) & $\mathrm{C}_{\mathrm{D}}(\mathrm{Numerical})$ \\
\hline 2.5 & 0 & 0 & 0.04 & 0.3979 & 0.3799 \\
\hline 2.5 & 1 & 0.1173 & 0.1222 & 0.4242 & 0.4064 \\
\hline 2.5 & 2 & 0.2943 & 0.3075 & 0.4526 & 0.4340 \\
\hline 2.5 & 3 & 0.4712 & 0.4938 & 0.4827 & 0.4614 \\
\hline 2.5 & 4 & 0.6482 & 0.6793 & 0.5148 & 0.5354 \\
\hline 2.5 & 5 & 0.8251 & 0.8655 & 0.5491 & 0.5721 \\
\hline 2.5 & 6 & 1.002 & 1.0470 & 0.5856 & 0.5598 \\
\hline 2.5 & 7 & 1.1791 & 1.1319 & 0.6245 & 0.5976 \\
\hline 2.5 & 8 & 1.3560 & 1.4224 & 0.6660 & 0.6374 \\
\hline 2.5 & 9 & 1.5331 & 1.4656 & 0.7103 & 0.7429 \\
\hline 2.5 & 10 & 1.7099 & 1.7851 & 0.7575 & 0.7903 \\
\hline 2.5 & 11 & 1.8869 & 1.9699 & 0.8079 & 0.7699 \\
\hline 2.5 & 12 & 2.0639 & 2.1506 & 0.8616 & 0.8271 \\
\hline
\end{tabular}

\section{$C_{L}$ vs Angle of Attack}

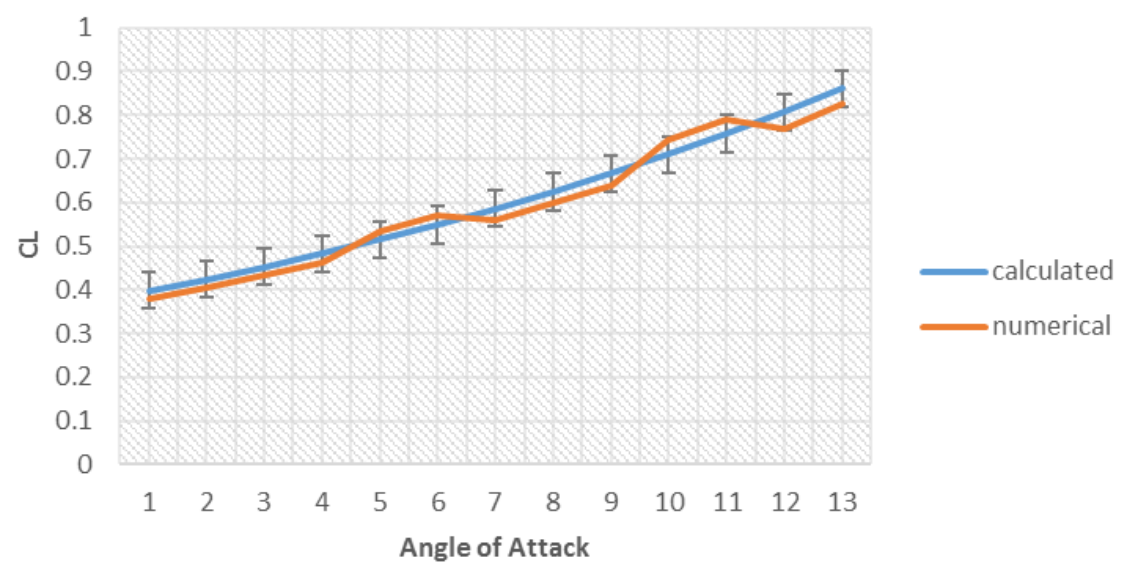

Figure 18. Plot of variation of numerical values of $C_{L}$ with Angle of Attack 


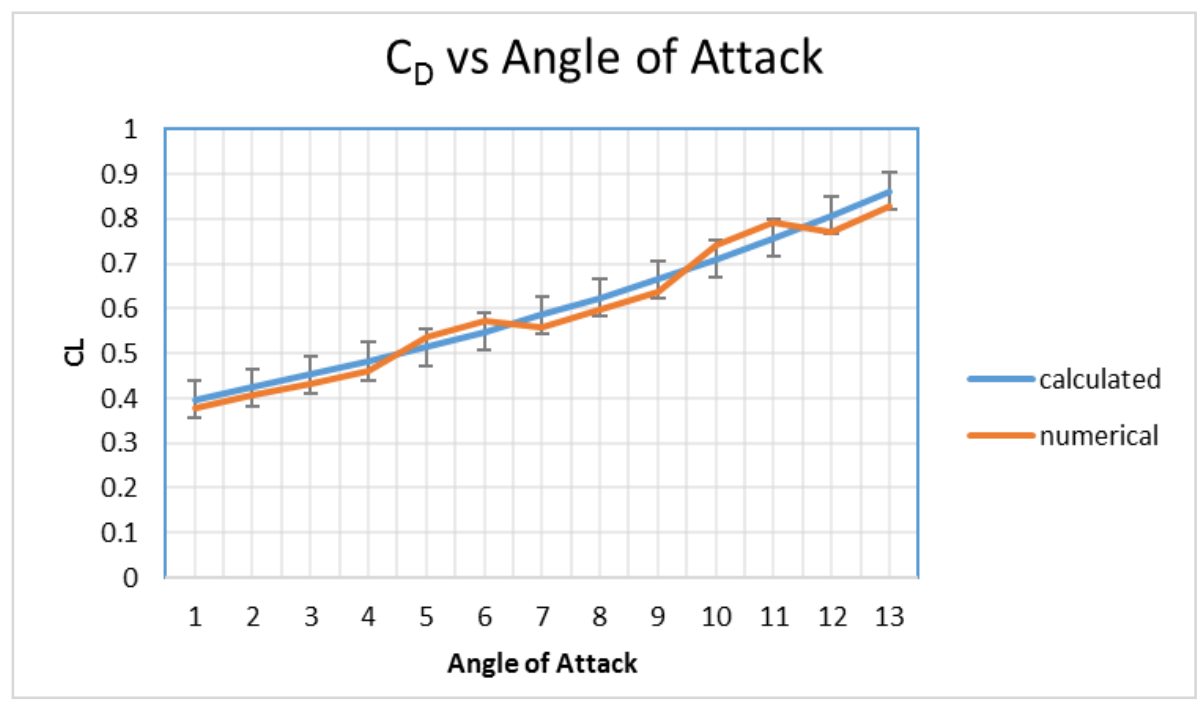

Figure 19. Plot of variation of numerical values of $C_{D}$ with Angle of Attack

The numerical values of $C_{L}$ and $C_{D}$ obtained from the numerical analysis performed were in close agreement with the calculated values as shown in fig 18 and 19.

\section{Conclusions}

From the numerical flow analysis over a Supersonic missile carried out on ANSYS Fluent to optimize the $C_{L} / C_{D}$ ratio by varying the angle of attack while keeping the Mach number same, it was found that the angle of attack giving the maximum $\mathrm{C}_{\mathrm{L}}$ is $12^{\circ}$ with a $\mathrm{CL}$ value of 2.15058 and with a maximum $C_{L} / C_{D}$ ratio of 2.5 .

As per the validation done using the results obtained from the analysis and the reference paper values, it was observed that the $C_{L}$ and $C_{D}$ values hold close agreement with each other within an error percentage of $4 \%$ to $5 \%$.

The shock waves formed due to the supersonic flow resulted in the decrease of the flow parameters such as velocity and Mach number, whereas increase in pressure, temperature and density values behind the shock wave.

With the increase in angle of attack, the shock waves generated become more intense and stronger and the flow separation at the end of the missile body also increases.

Increase in the $C_{D}$ value was found with increase in angle of attack from $0^{\circ}$ to $12^{\circ}$ which implied that the turbulence of the flow increase with angle of attack.

The maximum velocity obtained at the exit of the missile body also increased from angle of $0^{\circ}$ to $12^{\circ}$. Hence the aerodynamics efficiency of the missile, in the form maximum $\mathrm{C}_{\mathrm{L}} / \mathrm{C}_{\mathrm{D}}$ ratio was found to be maximum at an angle of attack of $12^{\circ}$ and hence it was inferred that the efficiency increases with increase in angle of attack of the missile.

\section{Acknowledgements}

We would like to thank our professor, Srinivas G., who had always been a great support and inspiration to us. Without his guidance, this paper wouldn't have been possible. Also, it was Armaan, a fellow student of Manipal Institute of Technology, who gave this paper the final touch and helped us through in the end.

\section{REFERENCES}

[1] Nenad Vidanovic, Bosko Rasuo, Gordana Kastratovic, Stevan Maksimovic, Dusan Curcic, Marija Samardzic, Aerodynamic-structural missile fin optimization, Aerospace Science and Technology 65 (2017) 26-45.

[2] Song Tian, Defu Lin, Jiang Wang, Bin Li, Dynamic stability of rolling missiles with angle of attack feedback three-loop autopilot considering parasitic effects, Aerospace Science and Technology 71 (2017) 592-60.

[3] Xinghui Yan, Jihon Zhu, Minchi Kuang, Xiangyang Wang, Aerodynamic shape optimizer using a novel optimizer based on machine learning techniques, Aerospace Science and Technology 86 (2019) 826-835.

[4] Li Heng, Ye ZhengYin, Effects of rotational motion on dynamic aero elasticity of flexible spinning missile with large slenderness ratio, Aerospace Science and Technology 95 (2019) 105384.

[5] Jacob Allen, Mehdi Ghoreyshi, Forced motions design for aerodynamic identification and modelling of a generic missile configuration, Aerospace Science and Technology 77 (2018) $742-754$.

[6] Yueyue Ma, Jie Guo, Shengjing Tang, High angle of attack command generation technique and tracking control for agile missiles, Aerospace Science and Technology 45 (2015) 324-334.

[7] Khizar Ahmed Pathan, P.S. Dabeer, Sher Afghan Khan, Optimization of area ratio and thrust in suddenly expanded flow at supersonic Mach numbers, Case Studies in Thermal 
Engineering 12 (2018) 696-700.

[8] Sachin Kumar, Shripad P. Mahulikar, Aero-Thermal analysis of lifting body configurations in hypersonic flow, Acta Astonautica, 126 (2016) 382-394.

[9] Armaan Aditya, Srinivas G "The Numerical Analysis of NACA 0018 Airfoil: Studying the Effect of Flap" International Journal of Mechanical and Production Engineering Reseach and Developments (IJMPERD). ISSN(P): 2249-6890; ISSN(E): 2249-8001, Vol. 9, Issue 4, Aug 2019, 1047-1054.

[10] Srinivas G, Madhu Gowda B P “Aerodynamic Performance Comparison of Airfoils by Varying Angle of Attack Using Fluent and Gambit" Journal of advanced materials research, vol. 592-594, 2014. pp 1889-1896. 